\title{
Fibromatosis Mesentérica: caso clínico-imagenológico y revisión bibliográfica
}

\author{
Carolina Whittle $\mathbf{P}^{1,2}$, Lorena Sánchez $\mathbf{R}^{2}$, \\ Juan Albarracin $\mathbf{G}^{3 a}$, Juan $\mathbf{H e p p} \mathbf{K}^{\mathbf{1}}$. \\ Retroperitoneal fibromatosis. \\ Report of one case
}

We report a 42 years old female that presented with abdominal pain and no palpable mass. Imaging abdominal ultrasound, CAT scan and magnetic resonance showed a solid tumor located in the retroperitoneum. The patient was operated on excising the tumor. Pathological examination of the surgical piece disclosed a retroperitoneal fibromatosis or desmoid tumor. Postoperative evolution of the patient was uneventful and she was discharged 12 days after the surgical procedure (Rev Méd Chile 2006; 134: 85-9).

(Key-words: Fibromatosis, abdominal; Retroperitoneal; Fibrosis)

Recibido el 27 de diciembre, 2004. Aceptado el 26 de julio, 2005.

${ }^{1}$ Servicio de Ecotomografía y Cirugía. Clínica Alemana de Santiago. ${ }^{2}$ Servicio de Radiología, Hospital San Juan de Dios. ${ }^{3}$ Facultad de Medicina Universidad de los Andes.

anterno de Medicina

L a fibromatosis mesentérica o tumor desmoides es un tumor de partes blandas del tipo fibroblástico localmente agresivo. Pese a ser una lesión benigna, que se origina en la fascia 0 en la aponeurosis muscular, tiende a infiltrar los tejidos adyacentes en forma radial. Es una lesión de presentación más bien esporádica, que se puede asociar al síndrome de Gardner, trauma o estados hiperestrogénicos. Los tumores desmoides se presentan con mayor frecuencia en pacientes adultos, en la cavidad abdominal, dependiendo de la pared abdominal, del mesenterio o del retroperitoneo. Para evitar la recurrencia local es importante detectar su localización y extensión en estadio pre-operatorio, para una adecuada planificación

Correspondencia a: Dra. Carolina Whittle. Servicio de Ecotomografía. Clínica Alemana de Santiago. Vitacura 5951. Vitacura. Fax 2101350. E-mail: cwhittle@alemana.cl de la resección. La presente comunicación describe el cuadro clínico y hallazgos de los exámenes por imagen de un paciente portador de fibromatosis mesentérica

\section{Caso CĹ́nico}

M.I.M.G., paciente de sexo femenino de 42 años. Con antecedentes de apendicectomía y hemioplastia inguinal derecha. Consultó por cuadro de dolor abdominal en noviembre de 2003. En estudio ecográfico se demostró nódulo hepático de $1 \mathrm{~cm}$. (probable hemangioma) y pólipo vesicular de $1 \mathrm{~cm}$. Persistió con dolor en hipocondrio derecho, por lo cual consultó nuevamente. Se solicitó ecotomografía que se realizó el 24 marzo 2004, que mostró tumoración del mesenterio de densidad adiposa, con imagen sugerente de linfonodos en su espesor, 
de $8,5 \times 3 \times 6 \mathrm{~cm}$. No se pudo descartar un proceso linfoproliferativo. La tomografía axial computada (TAC) abdominal (25.03.04), confirmó el pólipo vesicular de $1 \mathrm{~cm}$ y la existencia de dos hemangiomas hepáticos. Existía una tumoración sólida de la raíz del mesenterio, con algunos focos grasos en su interior, que estaba rodeada por asas intestinales. El tránsito intestinal confirmó el desplazamiento de las asas intestinales por un efecto de masa, sin alteración del relieve mucoso. El 26.03.04 la resonancia magnética mostró una masa tumoral sólida de la raíz del mesenterio, que sugeńa un tumor primario como primera posibilidad (linfoma, sarcoma, etc.).

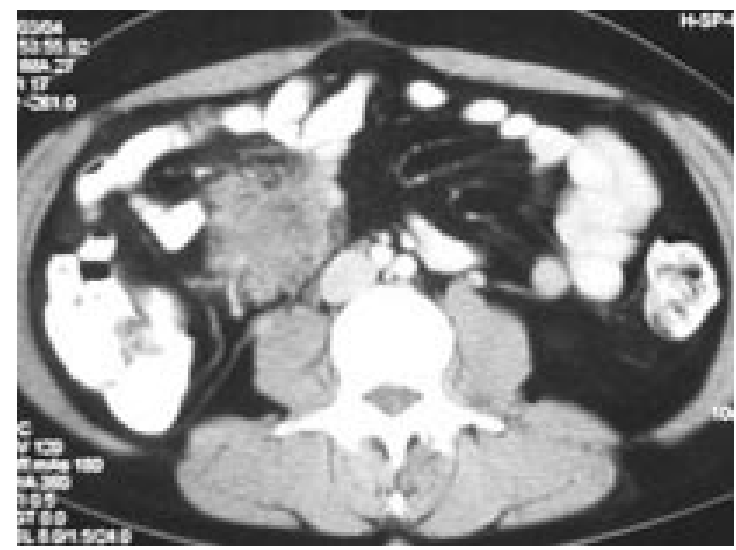

FIgURA 1. TAC muestra masa sólida en fosa iliaca derecha ubicada en la raíz del mesenterio de densidad algo heterogénea con pequeños focos de densidad grasa en su interior.

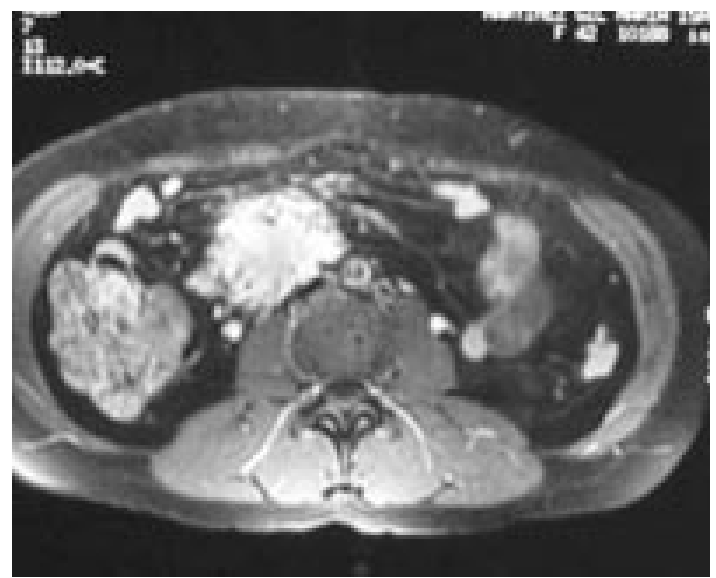

FIGURA 3. La resonancia magnética confirma masa tumoral sólida de la raíz del mesenterio.
Presentaba marcadores tumorales negativos (CA19-9, CA 125, $\alpha$ fetoproteína, CEA).

El 01.04.04 fue sometida a cirugía y en la exploración se detectó una lesión sólida del mesenterio del ileon distal de aproximadamente $9 \mathrm{~cm}$ de diámetro, que alcanzaba los vasos del mesenterio del intestino delgado e infiltraba el uréter derecho en aproximadamente $2 \mathrm{~cm}$. Existía un pólipo vesicular de $1 \mathrm{~cm}$. Se realizó resección de la masa retroperitoneal, del colon derecho, de $80 \mathrm{~cm}$ de ileon, colecistectomía y resección de $4 \mathrm{~cm}$ de uréter derecho con anastomosis término-terminal del uréter. Se

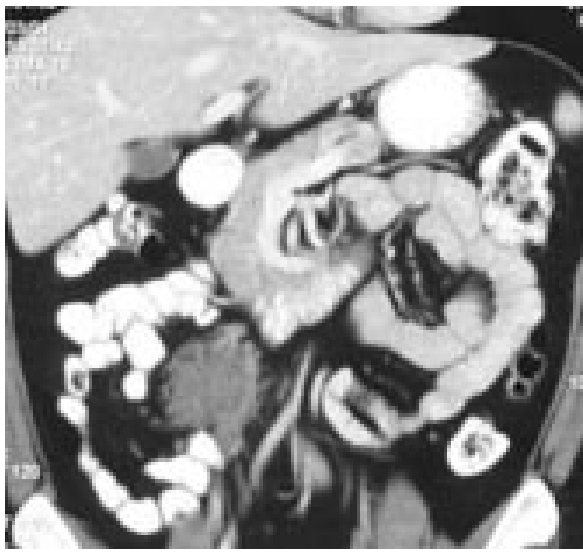

FIGURA 2. Reconstrucción de TAC permite ver la relación con las asas intestinales.

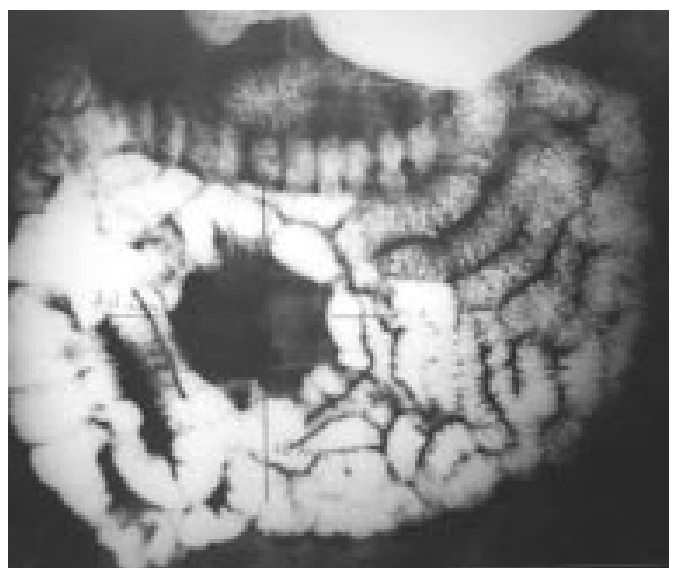

FIGURA 4. El tránsito intestinal confirma el desplazamiento de las asas intestinales por un efecto de masa sin alteración del relieve mucoso 

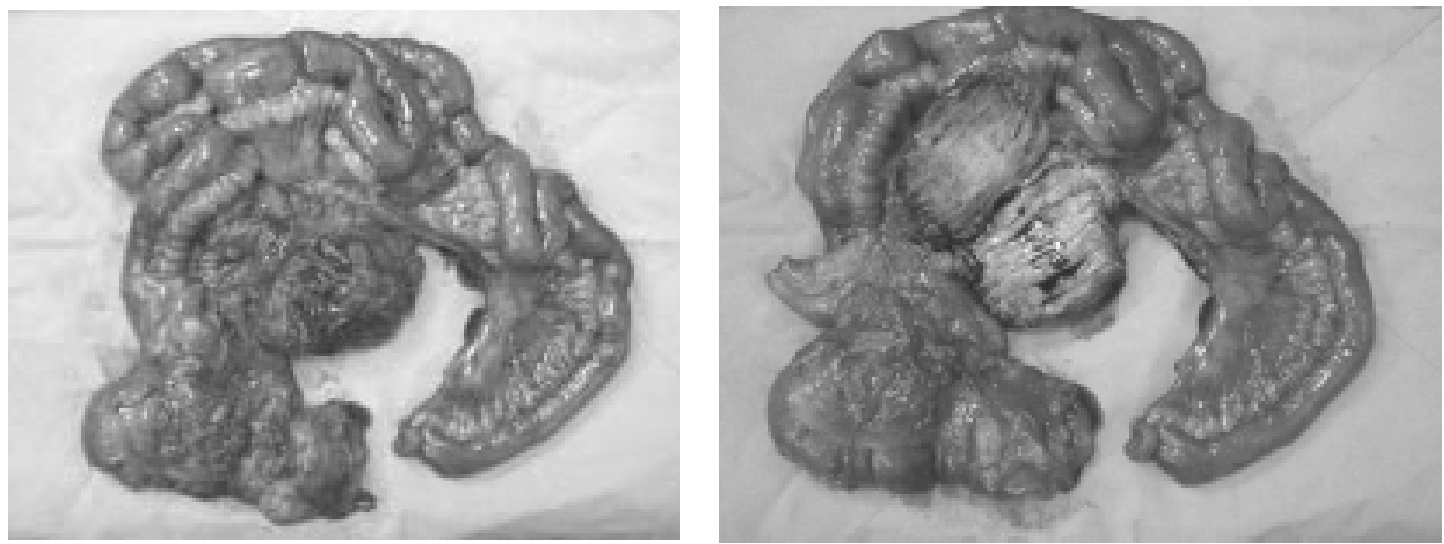

FIGURA 5 y 6 . Espécimen post resección de masa retroperitoneal y de $80 \mathrm{~cm}$. de ileon.

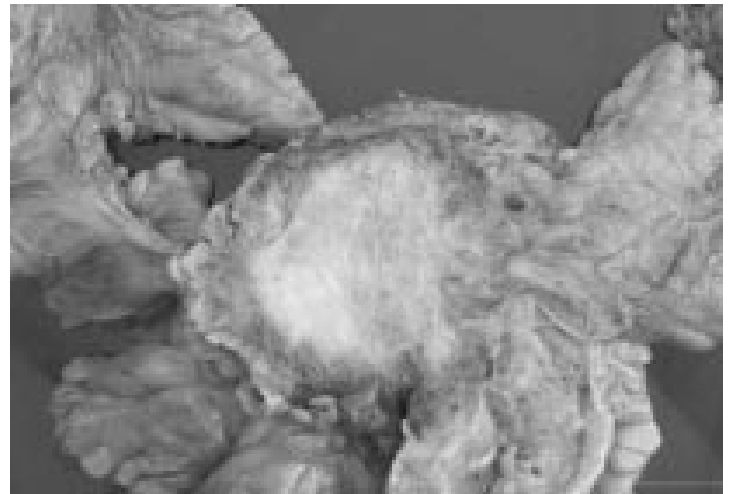

FIguRA 7. Macroscopia de la pieza quirúrgica. En el mesenterio se reconoce tumor de $8 \times 6 \times 4 \mathrm{~cm}$ de superficie pardo-violácea, con adherencias de tipo fibroso. Al corte es blanquesino-grisácea.

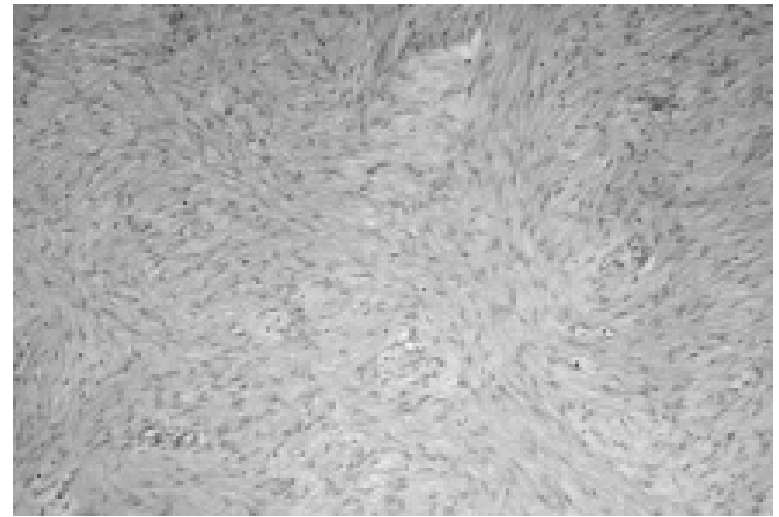

tomó biopsia rápida: tumor fibroso abdominal de carácter infiltrante.

Resultado de biopsia definitiva: Fibromatosis Mesentérica (Tumor Desmoide Intraabdominal). En el mesenterio se reconoció un tumor de $8 \times 6 \times$ $4 \mathrm{~cm}$, de superficie pardo-violácea, con adherencias de tipo fibroso. Al corte se vió blanquecinogrisácea, en parte fasciculada. Se ubicaba en la raíz del mesenterio. Microscópicamente estaba constituida por proliferación de células fusadas dispuestas en forma uniforme en un estroma colagenoso denso. Se observó muy escasas mitosis. La lesión infiltraba tejido adiposo del mesenterio, vasos y uréter derecho.

Biopsia vesicular: pólipos colesterínicos (2).

Evolucionó favorablemente en el postoperatorio, afebril, con buena cicatrización y sin compli-

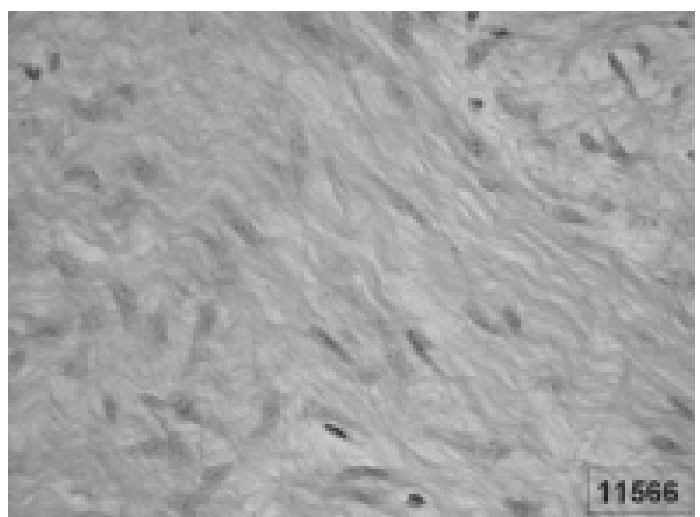

Figura 8 y 9. A microscopia está constituida por proliferación de células fusadas dispuestas en forma uniforme en un estroma de colágeno denso. Se observa muy escasa mitosis 
caciones evidentes. Se dio de alta el 13.4.04. Control alejado en julio de 2004, sin alteraciones.

\section{DisCUSIÓN}

La fibromatosis mesentérica o tumor desmoides es una lesión benigna de tipo fibroblástica que esta comprendida en el grupo de las fibromatosis. Por su crecimiento localmente agresivo con infiltración de los planos adyacentes, se ha definido en la clasificación de World Health Organization (WHO) como una lesión con grado de malignidad intermedia. Se origina en células fibroblásticas de la fascia o aponeurosis muscular.

La etiología del tumor desmoides no está clara, pero se han demostrado como factores etiológicos implicados en su desarrollo el trauma, alteraciones hormonales (hiperestrogenismo) ${ }^{2} \mathrm{y}$ factores hereditarios o genéticos (síndrome de Gardner) ${ }^{1}$, según las distintas publicaciones.

La fibromatosis abdominal se presenta con mayor frecuencia en adultos de sexo femenino, en la tercera o cuarta década de la vida, y se describe preferentemente en mujeres que han tenido 10 más hijos. Se localiza en la pared abdominal anterior (50\%), en el mesenterio (41\%) y en el retroperitoneo (9\%). Esto, a diferencia del desmoides extra-abdominal, que se ve con mayor frecuencia en hombres y cuyas ubicaciones más frecuentes son: dorso, pared torácica, cara y cuello y extremidad inferior.

Clínicamente, es una enfermedad poco frecuente, que se presenta en la mayoría de los casos en forma asintomática, pero con frecuencia existe un discreto dolor abdominal sin masa palpable. En algunos casos puede existir sangrado o abdomen agudo secundario a perforación de víscera hueca ${ }^{2}$. También puede dar complicaciones por la compresión ureteral como hidronefrosis ${ }^{4}$. Su diagnóstico es tardío, por lo cual es frecuente que al momento del diagnóstico tenga un tamaño aproximado a $10 \mathrm{~cm}$.

El diagnóstico por imágenes es de una tumoración retroperitoneal o interasas que se presenta al ultrasonido como una masa de contornos bien delimitados y que tiende a ser ecogénica por la presencia de grasa mesentérica ${ }^{3}$. Al TAC con uso de medio de contraste endovenoso, se aprecia como una tumoración sólida de coeficiente de atenuación mayor al del músculo y de márgenes bien 0 regularmente definidos ${ }^{1}$. A la resonancia magnética, se observa una masa de intensidad de señal menor a la del músculo en T1 y variable en las imágenes en $\mathrm{T}^{3}$.

Entre los diagnósticos diferenciales están:

El tumor carcinoide ${ }^{7,8}$ : es una neoplasia de las células endocrinas del intestino, que también puede originarse en pulmón, páncreas, tracto biliar, timo y ovario. Representa a 19\% de los tumores del intestino delgado. Se presenta como dolor abdominal vago e inespecífico. En 10\% de los pacientes hay un síndrome carcinoide por secreción excesiva de serotonina, prostaglandinas y quininas, lo que produce diarrea profusa, enrojecimiento, sudoración, dolor abdominal, hipotensión, insuficiencia cardíaca ventricular derecha, insuficiencia valvular tricuspídea o estenosis pulmonar por fibrosis endocárdica. Hallazgos en la TAC: masa de densidad de partes blandas, mal definida, infiltrativa. Puede asociarse a calcificaciones y reacción desmoplástica. También puede complicarse con isquemia y obstrucción. Cuando existen metástasis son hipervasculares.

Linfoma ${ }^{7}$ : Se presenta como una masa sólida a la ecotomografía y a la TAC, es muy rara la presencia de calcificaciones, salvo en estadio post tratamiento. Puede comprometer los vasos mesentéricos, pero es raro que presente complicaciones isquémicas. Post tratamiento puede simular una paniculitis mesentérica.

Esclerosis mesentérica ${ }^{8,9}$ : desorden inflamatorio del mesenterio, de etiología desconocida. Se asocia a otras entidades clínicas, como la fibrosis retroperitoneal, colangitis esclerosante, tiroiditis de Riedel, pseudotumor orbitario. Es más frecuente en sexo masculino, entre 20 y 90 años, como promedio, 60 años. Clínicamente da dolor abdominal, obstrucción o isquemia intestinal, masa abdominal o diarrea. En el laboratorio se describe VHS elevada y anemia. Su ubicación de elección es el mesenterio del intestino delgado. Cuando afecta al intestino grueso, lo hace preferentemente en el rectosigmoides. Puede coexistir con algunos tumores, entre ellos linfoma, cáncer de mama, pulmón, melanoma y colon. El estudio por imágenes varía de acuerdo al tejido predominante (grasa, tejido inflamatorio o tejido fibroso). En la TAC varía desde una sutil hiperdensidad del mesenterio hasta la presencia de una masa sólida 
de densidad de partes blandas. Puede asociarse a compromiso del porta hepatis y páncreas, vasos mesentéricos y desarrollo de vasos colaterales. Existe un signo característico ( fat ring sign») que traduce preservación de la grasa adyacente al vaso mesentérico comprometido. Puede existir calcificación en la porción central de la masa que traduce necrosis, también se han descrito formaciones quísticas y adenopatías mesentéricas $\mathrm{y}$ retroperitoneales.

Fibrosarcoma retroperitoneal ${ }^{6}$ : sólo $7 \%$ son retroperitoneales, su localización más frecuente es en las extremidades. Predomina en hombres en una razón de 2:1.

En la fibromatosis retroperitoneal el tratamiento de elección es el tratamiento quirúrgico con resección completa de la lesión. Si los márgenes son positivos o existe recidiva local se recomienda

\section{REFERENCIAS}

1. EINSTEIN DM, Taglabue JR, Desai RK. Abdominal desmoids: CT findings in 25 patients. AJR 1991; 157: 275-9.

2. BuRKe AP, SOBIN LH, SHEKITKA KM, FEDERSPIEL BH, HeIwIG EB. Intra-abdominal fibromatosis: a pathologic analysis of 130 tumors with comparison of clinical subgroups. Am J Surg Pathol 1990; 14: 335-41.

3. Casilas J, Sais GJ, Greve JL, Iparraguirre MC, MoRILO G. Imaging of intra- and extraabdominal desmoid tumors. RadioGraphics 1991; 11: 959-68.

4. BARON RL, LEE JK. Mesenteric desmoid tumors: sonographic and computed-tomographic appearance. Radiology 1981; 140: 777-9.

5. Kempson RL, FLETCher CDM, Evans HL, Hendrickson MR, SibLEy RK. Extra-abdominal fibromatosis (extra-abdominal desmoid fibromatosis). In: Kempson RL, Fletcher CDM, Evans HL, Hendrickson MR, Sibley RK, eds. Atlas of tumor pathology. I. Tumors of the soft tissues. Washington, DC: Armed Forces Institute of Pathology, 2001: 71-87.

6. VeRDECIA CANIZARES, CARIDAD. Fibrosarcoma retroperitoneal congénito. Rev Cubana Pediatr. abriljunio 2004. v 76; no2, p0-0.SIN 0034-7531. radioterapia adyuvante. En casos de irresecabilidad se debe plantear tratamiento radiante ${ }^{10,11}$. En aquellos casos rebeldes al tratamiento quirúrgico y radioterapia se recomienda el uso de quimioterapia y vitamina $\mathrm{D} 3^{12}$.

En conclusión, las fibromatosis en su totalidad corresponden a aproximadamente a $6 \%$ de los tumores benignos de partes blandas. Entre ellas está la fibromatosis profunda y de este subgrupo la ubicación retroperitoneal corresponde a aproximadamente $7,5 \%$.

Ante la existencia de una tumoración retroperitoneal se debe plantear entre los diagnósticos diferenciales el tumor desmoides, en especial si el paciente es de sexo femenino, entre la tercera y cuarta década de la vida. Los métodos de diagnóstico por imagen permitirán orientar al clínico en la formulación de un diagnóstico, pero no son específicos.

7. Sheth S, Horton KM, Gariand MR, Fishman EK. Mesenteric neoplasms: CT appearances of primary and secondary tumors and differential diagnosis. Radiographics; 2003 Mar-Apr; 23: 457-73.

8. Horton KM, Lawler LP, Fishman EK. CT Findings in sclerosing mesenteritis (panniculitis): spectrum of disease. Radiographics 2003; 23: 1561-67.

9. Poniachik TJ, Sмок SG. Mesenteris retráctril: Comunicación de cuatro casos. Rev Méd Chile 2000; 128: 1250-4.

10. Mendenhall WM, Zlotecki RA, MorRis CG, HoChWald SN, Scarborough MT. Aggressive fibromatosis. Am J Clin Oncol 2005; Apr 28: 211-5.

11. Micke O, Seegenschmiedt MH, German Cooperative Group on Radiotherapy for Benign Diseases. Radiation therapy for aggressive fibromatosis (desmoid tumors): results of a national Patterns of Care Study. Int J Radiat Oncol Biol Phys 2005 Mar 1; 61: 882-91.

12. Yildiz F, Kars A, Cengiz M, Yildiz O, Akyurek S, SeLEK U ET AL. 1,25-Dihydroxy vitamin D3: can it be an effective therapeutic option for aggressive fibromatosis. Med Hypotheses 2005; 64: 333-6. 